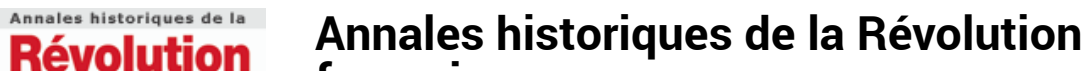

française française

\section{8 | avril-juin 2002}

La Révolution et le Droit

\section{Troubles populaires et société. Vues nouvelles sur l'Ancien Régime.}

À propos d'un livre récent

\section{Guy Lemarchand}

\section{(2) OpenEdition \\ Journals}

Édition électronique

URL : https://journals.openedition.org/ahrf/2254

DOI : 10.4000/ahrf.2254

ISSN : 1952-403X

Éditeur :

Armand Colin, Société des études robespierristes

Édition imprimée

Date de publication : 1 juin 2002

Pagination : 211-223

ISSN : 0003-4436

Référence électronique

Guy Lemarchand, «Troubles populaires et société. Vues nouvelles sur l'Ancien Régime. », Annales historiques de la Révolution française [En ligne], 328 | avril-juin 2002, mis en ligne le 11 mai 2006, consulté le 24 avril 2022. URL : http://journals.openedition.org/ahrf/2254 ; DOI : https://doi.org/ 10.4000/ahrf.2254

Ce document a été généré automatiquement le 24 avril 2022.

Tous droits réservés 


\title{
Troubles populaires et société. Vues nouvelles sur l'Ancien Régime.
}

\author{
À propos d'un livre récent *
}

\section{Guy Lemarchand}

1 On se souvient que c'est la querelle entre B. Porchnev et R. Mousnier ${ }^{1}$ qui, dans les années 1960, avait mis à l'ordre du jour de la recherche la question de la mesure et de la signification des troubles populaires dans la société française d'Ancien Régime. Le débat entre les deux historiens portait particulièrement sur la période du premier XVII ${ }^{\mathrm{e}}$ siècle antérieur au règne personnel de Louis XIV, mais les questions qu'il posait et les enseignements qu'on en tirait allaient bien au-delà de 1661 en amont et en aval et divers chercheurs, entre autres Y.M. Bercé ou E. Leroy-Ladurie ${ }^{2}$, ont repris et étendu les termes de la controverse dans la décennie 1970, d'ailleurs davantage dans l'inspiration de R.Mousnier que dans celle de Porchnev. Il semblait alors qu'après la révolte bretonne de 1675 et, en dehors de l'épisode essentiellement religieux des Camisards en 1702-1704, le gouvernement du Roi-Soleil avait maîtrisé la rébellion chronique grâce au développement de l'appareil répressif et à l'appui idéologique de la Contre-Réforme prêchant aux sujets du monarque l'obéissance. Ensuite, pensait-on, la croissance économique du xvIII ${ }^{\mathrm{e}}$ siècle, la poursuite, quoique ralentie, de la mise en place du réseau scolaire et le développement de l'assistance, si limitée qu'elle fut, avaient permis une relative pacification des mœurs populaires, en dépit d'une poussière de troubles de subsistances dus à la persistance de vieilles craintes irréfléchies en face de la circulation accrue de denrées. Ayant organisé une vaste enquête collective avec près de 60 collaborateurs, commencée en $1984^{3}$, portant sur la France dans ses frontières actuelles, utilisant l'informatique pour le recollement des fiches de dépouillement des sources, fiches établies au terme d'une longue discussion d'après une grille analytique large (24 séries de données éventuellement relevées), Jean Nicolas présente dans ce livre attendu la synthèse de l'énorme masse des renseignements recueillis. Ont été recensés non seulement les documents administratifs comme les lettres et rapports d'intendants, les mémoires des contemporains illustres et obscurs, mais surtout les sources judiciaires locales et supérieures, les délibérations municipales, les registres hospitaliers, la presse et, naturellement, la très vaste bibliographie des études 
historiques, aussi bien des érudits locaux anciens oubliés que les monographies et les travaux d'envergure nationale d'aujourd'hui. Il fallait une forte culture et une rigueur d'esprit affirmée pour dégager du sens du magma répétitif des faits mis à jour et l'auteur n'a manqué ni de l'une ni de l'autre. Une émeute isolée n'a en elle-même qu'une signification limitée, ce qui doit attirer particulièrement l'attention de l'historien, c'est la répétition des troubles, qui révèle un comportement durable et une pensée d'expression pas toujours aisée à décrypter mais réelle. Pour autant J.N. sait prendre une distance critique vis-à-vis de ce qu'il apporte. Ainsi il apparaît que les 8528 cas du corpus de l'enquête ne constituent qu'un minimum par rapport à la réalité, par exemple en matière d'Eaux et Forêts ou d'actions d'écoliers. Beaucoup n'ont donné lieu au maximum qu'à la rédaction de procès-verbaux sans aller plus loin par prudence ou faiblesse des autorités locales, papiers eux-mêmes disparus ensuite. De plus le discours des élites qui constitue la majeure partie de nos sources puisque les foules n'écrivent guère, tend selon les circonstances à majorer ou minimiser le nombre des manifestants qui seront nombreux quand l'administration veut amoindrir sa responsabilité ou au contraire ils seront quelques-uns seulement lorsqu'il s'agit d'endormir la suspicion du ministre auquel on s'adresse par conséquent les chiffres d'émeutiers sont fréquemment douteux. Dans le déroulement des faits les rumeurs peuvent jouer un rôle important, mais elles amplifient souvent abusivement les choses, tout en étant d'ailleurs en ellesmêmes significatives de la mentalité de leurs émetteurs.

2 En s'appuyant sur le vocabulaire assez varié et nuancé de l'époque, l'enquête avait retenu 72catégories de faits caractérisés par la participation d'au moins 4 individus et déployant une violence verbale ou physique contre les biens ou les personnes, regroupées en 13 ensembles. L'A. distingue l'intensité des troubles en fonction du nombre des manifestants (de 4 à 10,11 à 50 , plus de 50), les plus graves constituant près du quart du total. Il présente d'abord l'évolution globale. Après le calme relatif des années Colbert en dépit des pointes de l'Avènement et de 1675, la fin du règne est évidemment fortement agitée (1693, le sommet de 1709 [298 émeutes], ainsi que la Régence [1719-20, 1724]). Puis c'est l'apaisement Fleury rompu par la crise de 1740 (72 faits) et à partir de 1764 une montée presque continue de l'agitation avec des pointes attendues $(1768,1775)$ et une accélération encore de 1784 au 30avril 1789, date terminale de l'enquête (310 rébellions dans les quatre mois de 1789). $40 \%$ des faits ont lieu dans les villes de plus de 2000habitants. Viennent en tête des régions émeutières Paris et l'île-de-France, le Languedoc-Velay, la Bretagne et le Maine-Anjou, Alsace et Lorraine étant les plus paisibles. À l'origine de cette différenciation se trouvent à la fois les densités de population et, en sens contraire, l'implantation des forces militaires. Une objection vient immédiatement à l'esprit du lecteur en face de cette courbe annuelle l'augmentation du nombre des troubles n'est-elle pas due en fait à un meilleur enregistrement des événements par les autorités Contrairement à ce qu'on pourrait penser, en réalité les sources sont relativement abondantes et détaillées dès Louis XIV, et même il y en a plutôt davantage pour le règne du grand-roi avec les correspondances du Contrôle général et les papiers des Intendances que pour la fin du XVIII ${ }^{\mathrm{e}}$ siècle quant aux documents judiciaires, les fonds commencent fréquemment dès le milieu du XvII siècle ou même, pour les Cours Souveraines, avant.

3 Ensuite J. N. a rassemblé les ensembles de cas de l'enquête en 8 grands facteurs de base qu'il étudie successivement. Ce plan très analytique, malgré la répétition des scénarios de troubles d'un chapitre à l'autre, permet de saisir très précisément tous les aspects du 
phénomène de rébellion d'autant que, chemin faisant, l'A. relie toujours l'événement au contexte général lui-même évolutif, et l'on ne se lasse pas car le style acéré et vif de l'écriture reconstitue de façon particulièrement vivante ces scènes réjouissantes où le faible nargue le puissant et où le caquet inépuisable des grands se trouve un moment rabaissé. Les luttes antifiscales ne s'arrêtent pas avec la fin de Mazarin ou avec 1675 les rébellions contre les impôts indirects constituent $39 \%$ des cas pour toute la période et elles augmentent même (346 en 1661-1700, 1233 en 1761-1789). Si le Sud-Ouest est au premier rang, Paris même est touché. Rosser les gabelous pose son homme, même aux yeux des notables qui parfois s'y mettent également. Par contre la taille soulève de moins en moins la colère ( $3 \%$ des émeutes, seulement 16 en 1761-89) et même si elles provoquent un grand émoi chez les privilégiés et, dans les Parlements, force littérature apitoyée de défense « des peuples réduits à la plus extrême misère ", les nouveautés en matière de prélèvement direct, de la capitation au vingtième, ne heurtent guère la population, probablement à cause de leur apparence d'universalité. Viennent au deuxième rang, avec $14,1 \%$ des cas, les rébellions contre toute autorité, le guet, la maréchaussée, les soldats et les institutions et pratiques des forces de l'ordre, prisons, dépôts de mendicité, hôpitaux généraux, racolage, milice, c'est-à-dire tout ce qui représente une contrainte vécue comme une atteinte à la dignité. Les cas s'accroissent dans le premier tiers du XVIII ${ }^{\mathrm{e}}$ siècle et demeurent ensuite à peu près au même niveau. La seigneurie fait partie des cibles qui excitent les humbles, plus qu'on ne l'avait cru autrefois, et cela dès Louis XIV 67 cas de 1661 à 1689, 74 de 1690 à 1719, avec une apogée en 1780-1789 (122 cas) où se fait sentir la réaction seigneuriale qui n'est décidément pas un mythe. C'est la question des communaux qui mobilise le plus les paysans. Le XVIII ${ }^{\mathrm{e}}$ siècle est aussi par excellence le moment des troubles de subsistances 82 émeutes en 1740-1749, 334 en 1770-1779, 335 en 1780-1789, mais même si l'application des lois libérales sur le commerce des blés de 1763-1764 en multiplient les occasions, à toute époque le souci du pain est essentiel avec au total 17,6 \% des émeutes pour 1661-1789 et dès Louis XIV le trafic des grains soulève le peuple 22 émeutes en 1661-1669, 228 en 1700-1709. La participation des notables à ce type d'agitation est particulièrement rare et est mal vue des élites, car c'est le bien privé et non public qui est en cause. Parfois liés à la cherté des denrées mais souvent provoqués par l'embauche de salariés extérieurs au métier ou à la ville, les conflits du travail avec grèves apparaissent également plus fréquents qu'on ne le pensait, ce qui confirme et élargit ce qu'avaient déjà décrit $\mathrm{M}$. Garden, Ph. Minard et St.Kaplan ${ }^{4}$. Ils forment 5,1 \% du total des cas et vont croissant pendant la période, mêlant les rixes campagnonniques connus dès le début du XVII ${ }^{\mathrm{e}}$ siècle et, de plus en plus, les luttes contre les négociants entrepreneurs. Originalité de l'Ancien Régime et liée à des organisations spécifiques tolérées par les pouvoirs, les troubles provoqués par la jeunesse masculine ne constituent que 7,4 \% des cas, mais ils augmentent nettement après 1720 et plus encore après 1770. Ils visent fréquemment à maintenir des jeux et fêtes jugées impudiques ou insolents par les autorités et à rejeter ce qui relève de la discipline imposée d'en haut, ce qui fait écho aux rébellions de la dignité des adultes. Enfin, faiblement représentés avec 3,1\% du total, sont les désordres dus à la religion qui, seuls avec les affaires de la taille, déclinent après 1740-1760, les faits les plus nombreux étant suscités par l'opposition au Protestantisme de 1681 à 1720 , le jansénisme n'entraînant que peu d'incidents, essentiellement de 1720 à 1760 .

4 On regrettera seulement que l'A. ait écarté de son étude les troubles politiques parce que leur initiative et leurs thèmes viennent d'ailleurs que du populaire lui-même. 
Cependant les foules qui y participent peuvent avoir, malgré le caractère interclassiste et l'unanimité apparente de ces mouvements, leurs propres motivations et aucune organisation des élites n'est assez puissante et maîtresse en art de la manipulation psychologique pour les mettre en branle si elles n'y trouvent pas leur compte. On peut se demander également si les références religieuses justificatrices dans le discours des mutins ne sont pas aussi inexistantes que le suggère J.N. qui n'en parle pas n'y a-t-il pas une certaine influence de l'école élémentaire tenue par les clercs, comme l'a montré G. Ikni ${ }^{5}$ pour l'Oise pendant la Révolution et comme cela se produit dans l'Angleterre du $\mathrm{XVII}^{\mathrm{e}}$ siècle selon Ch. Hill ${ }^{6}$ L'Église ainsi que la littérature «bleue » des livrets et des almanachs $^{7}$ enseignaient le plus souvent la résignation aux humbles. Pourtant, dans la mesure où leur vision de l'univers n'avait pour source que cette connaissance d'un catéchisme simple, on sait depuis Engels que même le politique dans leur bouche était transposé en langage religieux fait d'images et de sentences d'origine biblique. Ici l'utilisation de certains concepts tirés de l'œuvre de P.Bourdieu (qui n'est pas cité dans la bibliographie générale alors qu'à juste titre un autre non-historien, M.Foucault, est mentionné et que sont données en marge supérieure de page des citations suggestives d'Héraclite et de Diderot) ne serait-elle pas utile, ainsi l'habitus, système de dispositions d'esprit permanentes, produit de l'enfance vécue et de la pratique sociale, qui oriente la perception qu'ont les individus de l'autre, ou bien encore la notion de «champ » en tant qu'ensemble invisible des relations entre groupes différents que noue une institution ou même une classe, particulièrement dans une société comme celle d'avant 1789 où la communication est encore assez rare et difficile à saisir Par ailleurs, si elle est liée à la croissance économique, la poussée des incidents pendant le XVIII ${ }^{e}$ siècle tient également à l'augmentation démographique relativement forte (28millions d'habitants dans l'hexagone en 1789 plutôt que 26 millions), au changement dans la pyramide des âges, aux mouvements migratoires amplifiés et à l'urbanisation, plus encore que ne le dit J.N. qui cependant n'oublie pas ce facteur dans ses explications.

5 Toutefois ces réserves de détail sont très secondaires par rapport à la richesse des apports de J.N. Le livre d'abord fait saisir de nombreux traits structurels majeurs et tendances de la société d'Ancien Régime. Tels sont, relevés au fil de la lecture, la pratique courante des déplacements à pied sur de longues distances qui facilite le recrutement des bandes de contrebandiers qui effectuent fréquemment des transports sur plusieurs centaines de kilomètres, le poids toujours agissant quoique mis en doute par quelques-uns aujourd'hui de la crise de cherté et de chômage selon le schéma d'E.Labrousse avec le mouvement des errants brutalement multipliés en marche vers les villes, le paternalisme seigneurial qui cherche à maintenir une suprématie devenue de plus en plus inutile et parasitaire, l'existence de groupes réputés dangereux qui vivent difficilement dans l'isolement des nombreux espaces vides de garrigues ou de forêts, l'écart entre d'une part la législation commerciale nouvelle et d'autre part la faiblesse des infrastructures de circulation qui ne sont que peu améliorées et la persistance des charges anciennes sur le mouvement des marchandises, l'inefficacité chronique des politiques d'assistance toujours détournées de leur but par le souci d'utilitarisme qui les anime aussi et, j'ajouterai, avec la grave insuffisance des moyens financiers qui y sont consacrés, les conflits de juridictions et la division entre les élites sans cesse renaissants qui freinent la répression. Surtout l'ouvrage bouleverse, avec prudence dans l'expression mais une grande sûreté dans les démonstrations, un certain nombre d'idées reçues. En premier lieu il conduit à nuancer singulièrement la vision d'un État monarchique rude manieur d'hommes et dévoreur de deniers mais 
innovateur progressiste imposant l'ordre et la justice aux foules enfermées dans leur ignorance, leurs réflexes élémentaires et leurs peurs ancestrales en face de tout changement. Certes il y a bien des traits d'archaïsme dans les mentalités populaires au $\mathrm{XVII}^{\mathrm{e}}$ et encore au $\mathrm{XVIII}^{\mathrm{e}}$ siècle, "champs privilégiés de la longue durée » avait déjà observé M.Vovelle. Ainsi dans les scènes décrites par J.N. apparaît d'abord l'irritabilité des mutins qui demeure constamment très vive et se mesure par exemple par l'écart entre la minceur du facteur déclenchant l'action et l'étendue de celle-ci. À Sète en 1781 il suffit d'une querelle de cabaret et de plaisanteries de soldats de la garnison à propos des consuls de la ville pour que commence une vaste bagarre faisant des blessés des deux côtés. La versatilité va de pair. À Cahors en 1779 alors que sont pendus deux assassins, profanateurs en outre de la croix, ce qui ne leur vaut certainement pas la sympathie de la foule, la corde cassant, le bourreau tente alors d'étrangler un des condamnés, mais le public se retourne et met en fuite l'exécuteur et les cavaliers de la maréchaussée qui maintenaient l'ordre. Bien que peut-être un peu atténuée au siècle des Lumières (J.N. en doute, faits à l'appui), la violence monte encore vite et peut devenir extrême. À Voultegon en Poitou en 1778 une bande de contrebandiers attaque au cabaret trois gabelous qui conduisent en prison deux des leurs et le sel saisi lors de l'arrestation, ils récupèrent le tout, tuent l'un des agents de la ferme et cassent bras et jambes aux deux autres. Il est vrai que la répression peut faire preuve d'une sauvagerie égale et on entre dans le cercle infernal provocation-répression qui sévit pendant tout l'Ancien Régime. L'État du XVIII ${ }^{\mathrm{e}}$ siècle se veut aussi « absolu » que celui du XVII ${ }^{\mathrm{e}}$ siècle et il n'hésite pas le cas échéant à se dresser brutalement contre le peuple au nom de l'ordre public et au service d'une réaction de classe de protection de la propriété. Lors de la fameuse émeute Réveillon à Paris en avril 1789 liée aux salaires, la troupe tire dans le tas, tuant plusieurs dizaines de manifestants, et la justice fait exécuter trois pendaisons quelques jours après. Autre trait d'archaïsme la superstition, ainsi la légende décrite par Condorcet et citée par J. N. du marchand de blé qui aurait été emporté par le diable, ou bien celle du pendu par le Parlement de Toulouse en 1748 à la suite d'un tumulte de subsistances qui serait devenu un saint parce que son corps exposé ne s'est pas corrompu. Le ritualisme conformiste accompagne aussi fréquemment l'action et lui donne une justification rassurante. À Henin-Liétard en Artois en 1784 le village se mobilise au son du tocsin contre les ouvriers venus labourer sur ordre du seigneur un marais jusque-là commun, et les femmes crient qu'elles ont toujours joui de cet espace (la référence au temps passé immémorial) et qu'elles n'obéiraient que sur un ordre du roi "bien signé par lui», papier que ne détient évidemment pas la maréchaussée présente. Par contre J. N. ne relève guère ce qui se pratiquait dans la première moitié $d u \mathrm{XVII}^{\mathrm{e}}$ siècle les jugements symboliques et dérisoires mis en scène par les mutins eux-mêmes des cibles de l'émeute, maltotiers, bladiers ou échevins, et l'évocation d'un âge d'or passé que bouleverserait l'arbitraire nouveau des autorités. Par contre, comme sous la Révolution après 1795 jusqu'au Xx siècle pour le souvenir vendéen en Vendée ${ }^{8}$, agit la mémoire collective qui fait qu'à partir de quelques événements rébellionnaires initiaux, certaines régions, le Languedoc, le Maine-Anjou qui ressortent des cartes de géographie statistique de J. N., demeurent longtemps plus sensibles et agitées que d'autres. Cet enracinement avait déjà été observé au XVII siècle par Y.M. Bercé pour le Sud-Ouest, de la Guyenne au Limousin. Les recherches de la démographie historique ${ }^{9}$ ont montré que les conditions de vie pour les catégories les plus basses des classes populaires, qui expliquent ces caractères psychologiques, ne s'étaient que peu améliorées au XVIII ${ }^{\mathrm{e}}$ siècle et même, 
comme le signale l'A., s'étaient à nouveau dégradées après 1770 . De plus si l'analphabétisme a reculé, l'illettrisme règne encore.

6 Cependant à ces traits anciens de mentalité s'en ajoutent d'autres, ambigus ou débouchant sur le changement. M. Vovelle avait déjà noté la sensibilité à certains événements et $C$. Jouhaud ${ }^{10}$ avait relevé qu'il n'y avait pas que répétition des comportements anciens du $\mathrm{XVII}^{\mathrm{e}}$ au $\mathrm{XVIII}^{\mathrm{e}}$ siècle. Avec l'étude de J.N. on voit que le communautarisme qui inspire nombre de troubles et se manifeste par la « rescousse » contre les gardiens de l'ordre, est particulièrement ambivalent. Souvent dans les affaires antifiscales, dans un sentiment d'unanimité de rejet de l'agression extérieure qui serait dirigée contre une certaine convivialité et bien-être des habitants, l'action unit des catégories sociales diverses, des pauvres jusqu'à quelques nobliaux ou le curé de la paroisse. Mais lorsqu'il s'agit de taxes municipales, cette même unanimité disparaît et le conseil de ville est vivement dénoncé. À Mézel en Provence en 1775 on s'en prend à «ces gens foutres de bourgeois " qui "veulent être les maîtres ", les consuls qui décident des octrois. Ainsi la communauté se restreint au plus grand nombre, le "bas peuple ", et on glisse vers l'expression d'un égalitarisme latent. Ce dernier trait est essentiel et quasiment constant en dépit du caractère interclassiste d'une partie des troubles recensés. Dans de nombreux cas concernant aussi bien l'impôt, les subsistances et le rejet de toute autorité qu'évidemment le travail artisanal et la seigneurie, les clivages sociaux apparaissent en cours d'action si ce n'est à ses débuts, et font monter la colère. Même la guerre des Camisards, qui pourtant $a$, bien sûr, une cause religieuse au premier chef, comporte un aspect social. Non seulement, comme le note l'A., parce que les combattants et les "prophètes » qui les dirigent se recrutent dans la petite paysannerie et les artisans ruraux et qu'il n'y a pas de gentilshommes parmi eux, mais également parce que, j'ajouterai, cette référence continuelle aux Écritures qui les anime est à la fois chargée de tradition calviniste et d'une marque d'égalité et de rejet des pompes du catholicisme hiérarchisé, propres à séduire ceux qui ne possèdent rien. Il en est de même du sens du point d'honneur et de la réclamation du respect de la dignité personnelle. Ils sont à la fois le résultat du mimétisme social, copier les dominants, la noblesse, comme en témoignent les multiples affaires de port illégal de l'épée, et joue également le refus du mépris venu d'en haut qui humilie la " populace », et s'exprime par là la revendication d'une liberté personnelle, une conscience libertaire dont on trouve aussi la trace dans des protestations de la jeunesse. Enfin l'existence quotidienne restant toujours précaire pour la masse des paysans parcellaires, journaliers et compagnons, s'affirme l'idée d'un droit naturel à la vie supérieur aux lois et interdits dictés par la société, ce que montrent les cris de défi et d'acceptation de la mort dans l'émeute, que ce soit la mort due aux fusils de la répression ou celle dictée par les jugements des tribunaux, car, comme il est dit à Beaufort en Maine-et-Loire en 1769, " autant périr la corde au col que de mourir de faim ». J.N., qui ne s'en explique pas, n'emploie pas le terme d'« économie morale " forgé par E.P. Thompson, mais il en retrouve l'intuition. Il serait du reste intéressant de comparer les enseignements tirés de cette étude des mouvements populaires aux résultats des recherches sur la criminalité d'Ancien Régime, en particulier à propos de la violence ${ }^{11}$. Au risque d'être accusé de ce que ne peuvent supporter les personnes de distinction, le misérabilisme, on peut dire que toute «novelté » qui est souvent en fait un prélèvement supplémentaire limité ou la privation d'un petit bien de consommation, pour maintes familles du populaire, est réellement intolérable. On a redécouvert récemment ce que savait déjà Thomas More, 
que les enclosures britanniques n'étaient pas nécessairement, malgré ce qu'en a dit plus tard A.Young, progressistes pour tous. Sans doute en est-il de même pour la hausse de l'impôt royal officiellement inégal et, en l'absence de progressivité, de la taille et des charges indirectes d'autant plus qu'il n'y a pas de mécanisme de redistribution vers le bas (mais il en existe vers le haut); ou peut également inquiéter l'accroissement de la circulation et du stockage des marchandises qui fréquemment en augmentent le prix dans l'immédiat. Le système économique des $\mathrm{XVII}^{\mathrm{e}}-\mathrm{XVIII}^{\mathrm{e}}$ siècles, en dépit de mercantilisme et des corporations, est en bonne partie réglé par le marché, au moins à l'échelle locale et régionale, et le marché n'a cure des bons sentiments. Est-il donc nécessaire d'invoquer encore ici l'archaïsme, la routine et le mythe lorsque l'on connaît la minceur et l'irrégularité des revenus populaires dans l'Ancien Régime Avec la faiblesse et la rigidité de beaucoup de salaires, l'étroitesse des rendements à la semence, le chômage qui arrive du jour au lendemain sans compensation, la moindre augmentation du coût des denrées due à la manipulation des aides, des octrois ou à une mauvaise récolte aggravée par la spéculation, ou bien même à la remise à jour de telle redevance pour le seigneur, peut être catastrophique, de même que la restriction de telle petite ressource secondaire liée à une réglementation ou une exclusion devenue soudain plus rigoureuse sur, par exemple, le droit à ramasser le bois à brûler qu'on tire du communal ou concernant la possibilité d'entretenir quelques têtes de petit bétail grâce à la vaine pâture. Tout cela est ressenti d'autant plus cruellement que le nombre absolu et souvent la proportion dans la population de ces titulaires de revenus précaires se sont accrus non seulement avec l'expansion démographique mais aussi avec l'accroissement des métiers battant du textile urbain, les progrès de la protoindustrie à la campagne, le développement des transports qui implique des charretiers comme des cabaretiers, etc., ce qui gonfle également les foules émeutières. Et dans le même temps, la crise séculaire $d u \mathrm{XVII}^{\mathrm{e}}$ siècle n'étant pas faite de cent ans de catastrophe continue, et la croissance économique du XVIII ${ }^{e}$ siècle étant réelle, au moins jusqu'à 1770 et pour certaines activités jusqu'à 1786 ou 1788, ainsi que la baisse des crises de mortalité après 1740 , le spectacle de l'inégalité est devenu plus évident et provocateur avec le développement du luxe du vêtement, la multiplication des carrosses, la construction des hôtels de négociants à la ville ou, grâce à la hausse des fermages ou du produit des dîmes, la réfection de châteaux ou des bâtiments monastiques au village et l'édification des maisons de briques des gros fermiers. De même qu'on pouvait s'enrichir sous Louis XIV grâce entre autres à l'exportation des toiles ou au trafic des finances du roi ou en rachetant les terres des parcellaires appauvris par les chertés, la "douceur de vivre » du XvIII ${ }^{\mathrm{e}}$ siècle existe bien mais elle n'est pas pour tout le monde, surtout avec la décélération d'après 1760-1770 de diverses industries et du commerce. On sait bien que l'économique et le social sont non pas isolés l'un de l'autre, comme dans les livres d'historiens pour la clarté de l'analyse si ce n'est quelquefois par le formalisme d'une pensée attachée à la pureté et l'indépendance des catégories, ils sont vécus en même temps par les contemporains et réagissent l'un sur l'autre et réciproquement

7 Par conséquent dans la mentalité populaire et de façon variable suivant les circonstances coexistent contradictoirement l'ancien et le nouveau, le conformisme et la critique sociale, voire une pensée prépolitique que l'on trouve par exemple dans le thème de pacte de famine, mais avant même l'épisode de la cabale parlementaire de 1768 , sous forme de dénonciation de la complicité des autorités locales avec les «partisans » ou les «monopoliers ». Et le « complot» des cours souveraines ou de la 
grande noblesse n'est pour rien dans la guerre des farines, mais l'élargissement du thème infamant d'une alliance entre le négoce céréalier et les administrateurs jusqu'au cercle de l'entourage royal marque un début de désacralisation de la monarchie. La passivité est loin d'être la règle, et s'il faut souligner avec R.Muchembled ${ }^{12}$ le poids de la culture des élites sur les humbles, pour autant la culture d'origine populaire - les croyances mythiques et les pratiques ritualisées marquées par le naturalisme, l'animisme et le magisme - n'a pas totalement disparu au $\mathrm{xVIII}^{\mathrm{e}}$ siècle comme le font voir les manifestations de jeunesse, elle contribue à entretenir à la fois le communautarisme et le liberteranisme qui les inspirent et elle est même si vivante encore qu'on la retrouve, bien qu'amoindrie, au siècle suivant, ainsi que l'a établi A.Van Gennep ${ }^{13}$. Dans la même ligne B.Garnot avait déjà relevé qu'il y avait une autonomie croissante du populaire par rapport aux élites dans le domaine du vêtement et on peut noter également que se développe la lecture à Paris ${ }^{14}$, sans parler d'un début de liberté sexuelle signalé par la multiplication des naissances illégitimes et des conceptions prénuptiales, parallèles à la baisse de la ferveur et du conformisme religieux, décléricalisation ne signifiant pas pour autant déchristianisation au sens littéral du mot. Cette dernière remarque nous met sur la piste d'une autre idée-force de l'ouvrage de J.N. Sous l'influence de Tocqueville nous tendons à penser que les idées nouvelles descendent des cercles philosophiques vers les basses couches sociales, elles les auraient réveillées et poussées au soulèvement, mouvement qui aurait été l'une des origines de la secousse de la Révolution. Or à propos de la critique des droits seigneuriaux par la Physiocratie et Boncerf, ou à propos du libéralisme industriel de Gournay et Turgot, ou encore, avec les protestations de Voltaire ou Beaumarchais contre les châtiments infligés aux laquais, il apparaît que le refus des institutions et pratiques anciennes autoritaires et violentes vis-à-vis du peuple est d'abord venu d'en bas. En effet la continuation des exigences et disciplines traditionnelles s'est heurtée à une opposition de plus en plus étendue et les écrivains et publicistes ont plutôt théorisé un mouvement qu'ils ne l'ont lancé. Néanmoins l'énoncé des principes et projets novateurs renforce à son tour l'action des foules en les justifiant en droit, comme le montre la pénétration du vocabulaire philosophique dans les cahiers de doléances.

8 Le livre nous oblige encore à repenser d'autres questions qui sont liées au mouvement global rébellionnaire pendant l'ensemble de la période. Il conduit d'abord à compléter la réévaluation du règne de Louis XIV menée par P.Goubert ${ }^{15}$ et à relativiser encore plus le caractère absolu de... l'«absolutisme » du Roi-Soleil. En fait, en dépit de la grandeur artistique et intellectuelle du clacissisme dominant et de l'activité réelle de création législative et institutionnelle du régime, celui-ci est loin de maîtriser totalement la situation sociale et il y a sur ce plan continuité avec le moment Mazarin. Évidemment l'État est consolidé au fil des décennies suivantes puisque effectivement après 1675 disparaissent les révoltes à l'échelle de plusieurs élections ou plusieurs provinces. Néanmoins l'agitation populaire n'est pas éteinte pour autant et on voit que même les Protestants qu'on pouvait croire découragés par les mesures de compression qui se succèdent de 1663 à 1685, résistent, particulièrement les adhérents des classes inférieures, et on compte encore 12 échauffourées entre eux et l'armée depuis la Révocation jusqu'au soulèvement Camisard. De plus comme les guerres fréquentes obligent le gouvernement à accroître les ponctions d'argent et même d'hommes (milice et classes de marine) et qu'il agit avec une totale brutalité hautaine, la misère et le mécontentement explosent après 1690 . Quant à l'évolution pendant le XVIII ${ }^{\mathrm{e}}$ siècle, il apparaît à l'évidence que la Révolution n'est pas déclenchée seulement par la révolte 
nobiliaire en 1787-1788 ni par la diffusion des principes philosophiques. Les grands débats politiques autour de la convocation des états généraux et des réformes à entreprendre ont été précédés par une effervescence populaire qui va croissante depuis près d'un demi-siècle. Si les élites et les autorités demeurent longtemps sourdes au discours d'en bas, le peuple enfant ne pouvant dans ses tumultes, selon elles, qu'être l'instrument des intrigues des ambitieux, elles n'en subissent pas moins cette pression qui les amène à céder partiellement et par à-coups, ce qui ne résout rien durablement et contribue à faire monter un peu plus la colère. S'agit-il d'une vue téléologique des événements des dernières années de l'Ancien Régime Les chiffres annuels de troubles et plus encore le comptage par quinquennats parlent d'eux-mêmes 307 incidents en 1760-1764, 869 en 1785-1789 (4mois). Surtout il faut relire les remarques des administrateurs et des observateurs divers de la seconde moitié du xvIII ${ }^{e}$ siècle qui relèvent la diffusion parmi les manifestants et les paysans d'un esprit d'« insolence " vis-à-vis des membres de la hiérarchie sociale, en particulier des seigneurs, ou l'esprit d'« indépendance » des ouvriers urbains, ou encore l'esprit «républicain» (J. N. a compté plus de 50 occurrences de ce terme, toujours à connotation péjorative parce qu'il met en cause la hiérarchie) chez les montagnards des Vosges arguant en 1744 de leur droit sur la forêt communale contre l'abbaye de Munster et le Magistrat de la ville. En même temps se raréfient les alliances interclassistes qui se formaient auparavant dans certaines émeutes entre petit peuple et notables et qui du reste étaient souvent assez éphémères, et bourgeois et nobles qui laissaient avec bienveillance se dérouler les rituels populaires folkloriques, cessent désormais d'y participer. Tend également à se répandre l'idée que le monde du travail constitue un groupe à part, préface des " classes dangereuses » du xix siècle. Les " gens de peu ", devenus plus nombreux avec la croissance démographique et plus remuants, inquiètent et la tension sociale se développe.

9 À la lumière de cette étude sur 1661-1789 et de sa méthode, il faudrait réexaminer aussi systématiquement d'autres périodes qualifiées peut-être sommairement de calmes. Ainsi pour la fin $d u \mathrm{Xv}^{\mathrm{e}}$ siècle et la première moitié $\mathrm{du} \mathrm{XVI}^{\mathrm{e}}$ siècle, depuis la régence des Beaujeu après les séditions antifiscales du règne de Louis XI jusqu'à 1560, période qui n'aurait connu que la Grande Rebeyne de Lyon (1529) et le soulèvement du Sud-Ouest de $1548^{16}$. De même pour la Révolution nous n'avons d'enquête étendue que pour les campagnes de 1789 à $1794^{17}$ et s'offre un autre moment réputé apaisé de 1796 à 1811, avant les troubles de 1812. Peut-être l'écroulement rapide de l'Empire en 1814, renouvelé à la fin des Cent-Jours, serait-il plus facile à comprendre si nous saisissions mieux les réactions populaires des années 1810. Le beau livre de Jean Nicolas devrait donc faire école.

\section{NOTES}

*.Jean NICOLAS, La rébellion française. Mouvements populaires et conscience sociale 1661-1789, Le Seuil, Paris, 2002, 620 pages. 
1.Boris PORCHNEV, Les soulèvements populaires en France de 1623 à 1648, Paris, 1948, trad. française, 1963 Roland MOUSNIER, « Recherche sur les soulèvements populaires en France avant la Fronde ", Revue d'Histoire moderne et contemporaine, 1958, 2 du même, Fureurs paysannes. Les paysans dans les révoltes du XVII siècle (France, Russie, Chine), Paris, 1967. 2.Yves-Marie BERCÉ, Histoire des Croquants. Étude des soulèvements populaires au XVII siècle dans le Sud-Ouest de la France, 2 vol., Genève - Paris, 1974 du même, Fête et révoltes. Des mentalités populaires $d u \mathrm{XVI}^{e}$ au XVIII ${ }^{e}$ siècle, Paris, 1976 Emmanuel LE ROY-LADURIE, « Révoltes et contestations rurales en France de 1675 à 1789, Annales ESC, 1974, 1 du même, Le carnaval de Romans 1579-1580, Paris, 1979, nuance quelque peu l'article précédent.

3.J. Nicolas avait dirigé un important colloque qui est à l'origine de l'enquête Mouvements populaires et conscience sociale $\mathrm{XVI}^{e}$-XIX ${ }^{e}$ siècles, Paris, 1985.

4. Maurice GARDEN, Lyon et les Lyonnais au XVIII ${ }^{e}$ siècle, Paris, 1970 Philippe MINARD, La fortune du colbertisme. État et industrie dans la France des Lumières, Paris, 1998 ST.KAPLAN, La fin des corporations, Paris, 2001.

5.Guy IKNI, Crise agraire et révolution paysanne. Le mouvement populaire dans les campagnes de l'Oise de la décennie physiocratique à l'An II, Thèse, Paris I, 1993, dactyl.

6.Christopher HILL, Collected essays, t. III People and ideas in XVIIe century England, Brighton, 1986. Cf. aussi E.P. THOMPSON, « L'économie morale de la foule dans l'Angleterre du XVIII ${ }^{\mathrm{e}}$ siècle, dans La guerre du blé au XVII siècle, Paris, 1988.

7.Robert MANDROU, De la culture populaire aux XVII et XVIII ${ }^{e}$ siècles. La Bibliothèque bleue de Troyes, Paris, 1964, 2e éd. revue 1975 Geneviève BOLLÈME, Les almanachs populaires aux XVII et XVIII ${ }^{e}$ siècles. Essai d'histoire sociale, Paris, 1969 Pierre GOUBERT, Daniel ROCHE, Les Français et l'Ancien Régime, 2 vol. t. II, Paris, 1984.

8.Jean-Clément MARTIN, La Vendée, la mémoire 1800-1980, Paris, 1989.

9.Scarlett BEAUVALET-BOUTOUYRIE, La démographie de l'époque moderne, Paris, 1999.

10.Michel VOVELLE, Idéologie et mentalités, Paris, 1982 Christian JOUHAUD, dans dir. A.BURGUiÈRE, J. REVEL, Histoire de la France, t. III; L'État et les conflits, Paris, 1990.

11. Yves CASTAN, Honnêteté et relations sociales en Languedoc 1715-1780, Paris, 1974 NicolecASTAN, Les criminels du Languedoc. Les exigences d'ordre et les voies du ressentiment dans une société pré-révolutionnaire 1750-1789, Toulouse, 1980 dir. Benoît GARNOT, Histoire et criminalité de l'Antiquité au XX $X^{e}$ siècle, Dijon, 1992 Benoît GARNOT, Crime et justice aux XVII et XVIII ${ }^{e}$ siècles, Paris, 2000.

12.Robert MUCHEMBLED, L'invention de l'homme moderne. Sensibilités, mœurs et comportements collectifs sous l'Ancien Régime, Paris, 1988. Dans cet ouvrage R. Muchembled revient sur le thème de l'acculturation écrasante des couches populaires et l'abandonne partiellement par rapport à ce qu'il avait écrit dans Culture populaire et culture des élites dans la France moderne XV'-XVIII siècle, Essai, Paris, 1978.

13.Arnold VAN GENNEP, Manuel de folklore français contemporain, 9 vol., Paris, 1937-1958. 14.Benoît GARNOT, Le peuple au siècle des Lumières. Échec d'un dressage culturel, Paris, 1990 Daniel ROCHE, Le peuple de Paris. Essai sur la culture populaire au XVIII ${ }^{e}$ siècle, Paris, 1981 pour le vêtement, cf. D. ROCHE, La culture des apparences. Une histoire du vêtement XVII ${ }^{e}-X V I I I^{e}$ siècle, Paris, 1989.

15.Pierre GOUBERT, Louis XIV et vingt millions de Français, Paris, 1966, rééd. revue 1991. 16. Roger GASCON, Grand commerce et vie urbaine au XVIe siècle. Lyon et ses marchands, 2 vol., Paris, 1971 Yves-Marie BERCÉ, Croquants et Nus-Pieds. Les soulèvements paysans en France du 
$\mathrm{XVI}^{e}$ au XVIII $I^{e}$ siècle, Paris, 1974 Guy LEMARCHAND, « Troubles et révoltes populaires en France $\mathrm{XVI}^{\mathrm{e}}-\mathrm{XVII}{ }^{\mathrm{e}}$ siècles, dans Féodalisme, société et Révolution française. Études d'histoire moderne XVI ${ }^{e}$-XVIII siècle, Caen, 2000.

17.Anatoli ADO, Paysans en Révolution. Terre, pouvoir et jacquerie 1789-1794 (1987), trad. Paris, 1996. 